\title{
Combination of oncolytic adenovirus ZD55 harboring TRAIL-IETD- MnSOD and cytokine-induced killer cells against lung cancer
}

\author{
Runde Jiang, Zhixiong Zhang, Xinghai Liao, Liangjuan Huang, Yilang Liao, Weiyi Deng \\ Department of Pathology, Shenzhen Hospital of Southern Medical University, Shenzhen, China \\ Contributions: (I) Conception and design: R Jiang; (II) Administrative support: R Jiang; (III) Provision of study materials or patients: R Jiang, Z Zhang, \\ X Liao; (IV) Collection and assembly of data: R Jiang, L Huang, Y Liao; (V) Data analysis and interpretation: R Jiang, W Deng; (VI) Manuscript \\ writing: All authors; (VII) Final approval of manuscript: All authors. \\ Correspondence to: Dr. Runde Jiang. Department of Pathology, Shenzhen Hospital of Southern Medical University, 1333\# Xinhu Road, Bao'an \\ District, Shenzhen 518000, China. Email: rundej@126.com.
}

\begin{abstract}
Background: Our study aimed to investigate the effect of cancer-targeting gene-virotherapy and cytokineinduced killer (CIK) cell immunotherapy on lung cancer.

Methods: CIK cells were obtained from peripheral blood mononuclear cells using interferon (IFN)- $\gamma$, interleukin (IL)-2, and CD3 monoclonal antibody. The CIK cells were infected with oncolytic adenovirus ZD55 harboring tumor necrosis factor (TNF)-related apoptosis-inducing ligand (TRAIL), manganesecontaining superoxide dismutase (MnSOD), and TRAIL-isoleucine-aspartate-threonine-glutamate (IETD)MnSOD. The cells were then cocultured with lung cancer cell lines A549 and NCI-H1650, normal cell line BEAS-2B, or injected into an A549 xenograft mouse model.

Results: Proliferation, colony formation, and invasion of A549 and NCI-H1650 cells were significantly inhibited by co-cultivation with CIK cells carrying oncolytic adenoviruses (in order) ZD55-TRAIL-IETDMnSOD > ZD55-TRAIL + ZD55-MnSOD > ZD55-MnSOD > ZD55-TRAIL. Compared to BEAS-2B cells, the production of IFN- $\gamma$, TNF- $\alpha$, and lactate dehydrogenase (LDH) in tumor cells was increased. Tumor volume in the xenograft model and Ki-67 expression in tumor samples were reduced after injection of CIK cells carrying oncolytic adenoviruses, in the same order as the in vivo experiments. Levels of IFN- $\gamma$, TNF- $\alpha$, and LDH contents were also increased in the same order.

Conclusions: Our studies confirmed the high efficacy of combined oncolytic adenovirus ZD55 harboring TRAIL-IETD-MnSOD and CIK cells against lung cancer.
\end{abstract}

Keywords: Cytokine-induced killer cells; lung cancer; xenograft model; oncolytic adenovirus

Submitted Jul 30, 2021. Accepted for publication Oct 18, 2021.

doi: 10.21037/atm-21-4479

View this article at: https://dx.doi.org/10.21037/atm-21-4479

\section{Introduction}

Lung cancer is one of the foremost causes of cancer-related deaths worldwide. The 5 -year survival rate ranges from approximately $20 \%$ to $75 \%$ (1-4) due to drug resistance, genetic mutation, and cancer recurrence $(3,5)$. Researchers are focusing on these challenges and exploring new therapeutic strategies for improving patient survival rates and the clinical outcomes of lung cancer.

Cancer-targeting gene-virotherapy (CTGVT) is a novel combined strategy of gene and virus therapies that is currently being explored. CTGVT has been widely reported to have high antitumor efficacy in in vivo animal tumor models and cytopathic efficacy in in vitro cancer cells $(1,6-8)$. Oncolytic viral vectors, or oncolytic adenoviruses, have anticancer capacity due to their gene carrying ability, broad range of cell targets, capacity to lyse cancer cells, selective replication in cancer cells, and few side effects $(8,9)$. Oncolytic adenoviruses have been 
found to replicate in cancer cells and not in normal cells. Anticancer activity is increased in oncolytic adenoviruses harboring apoptosis-inducing gene tumor necrosis factor (TNF)-related apoptosis-inducing ligand (TRAIL) (6) and tumor suppressor genes, including manganese-containing superoxide dismutase (MnSOD) (10), second mitochondriaderived activator of caspase (Smac) (11), and inhibitor of growth 4 (ING4) (6). The preferential replication of oncolytic viral vectors in cancer cells ensures the high expression of these tumor suppressors, thereby inhibiting tumor growth and even leading to eradication. However, the tumor specificity of oncolytic adenoviruses in lung cancer cells is not high (8).

Another novel therapy for treating solid tumors is infusion of cytokine-induced killer (CIK) cells, the immune-active host effector cells with the major histocompatibility complex (MHC)-restricted cytotoxicity of killing cancer cells $(12,13)$. CIK cell strategy is an alternative immunotherapy aimed at improving the body's immune response to cancers (14). The administration of interferon (IFN)- $\gamma$ and interleukin (IL)-2 increases CIK cell numbers, and in return, CIK cells secrete inflammatory cytokines, including IFN- $\gamma$, IL-2, interleukin (IL) -10 , and TNF- $\alpha(12,15,16)$. This therapy has been widely applied in the treatment of various cancers, including breast cancer (13), renal cell carcinoma (14), and lung cancer (17), with studies showing that the use of autologous CIK cell therapy improved overall survival rates of cancer patients $(15,17)$. The combination of CIK cell therapy with CTGVT in human cancers is capable of higher efficacy than single treatments and is being extensively tested worldwide $(18,19)$. However, there is little information on the efficacy of combined therapeutic treatment in lung cancer.

This study was the first time to investigated the efficacy of combined CIK cell therapy and oncolytic adenovirus ZD55 harboring TRAIL and MnSOD in lung cancer cells by establishing CIK cells carrying the oncolytic adenovirus ZD55 harboring TRAIL-isoleucine-aspartate-threonineglutamate (IETD)-MnSOD gene and then exploring the in vitro and in vivo efficacy of this combined therapy in lung cancer. In this study, CIK cells were induced and then reformed with oncolytic adenovirus ZD55 harboring tumor necrosis factor (TNF)-related apoptosis-inducing ligand (TRAIL), manganese-containing superoxide dismutase (MnSOD), and TRAIL-IETD-MnSOD. Finally, the CIKs was then co-cultured with lung cancer cell lines A549, NCI-H1650 and normal cell line BEAS-
$2 \mathrm{~B}$, or injected into A549 xenograft mouse model, to investigated the anti-cancer effect of CIKs. This study provided novel insights into the therapy as well as more data on the combined application of CIK therapy and CTGVT in human cancers. We present the following article in accordance with the ARRIVE reporting checklist (available at https://dx.doi.org/10.21037/atm-21-4479).

\section{Methods}

\section{Cells and cell culture}

Peripheral blood $(10 \mathrm{~mL})$ samples were collected from lung cancer patients. Peripheral blood mononuclear cells (PBMCs) were separated from the samples using PBMC isolation solution (Shanghai Solarbio Bioscience and Technology Co., Ltd., Shanghai, China). PBMCs were counted, separated by centrifugal precipitation, suspended in PBS buffer containing $0.5 \%$ bull serum albumin (BSA) (Solarbio), and incubated with CD14-conjugated magnetic beads (Miltenyi Biotec, Auburn, CA, USA) at $2-8{ }^{\circ} \mathrm{C}$ for 15 minutes, followed by resuspension and centrifugation. After being washed 3 times, the peritoneal cell-derived mast cells (PCMCs) were eluted, collected, and then incubated in Iscove's Modified Dulbecco's Medium (IMDM, pH 7.2-7.4; GIBCO-BRL, Grand Island, NY, USA) supplemented with $10 \%$ fetal bovine serum (FBS) and $100 \mathrm{U} / \mathrm{mL}$ penicillin-streptomycin at $37{ }^{\circ} \mathrm{C}$ with $5 \% \mathrm{CO}_{2}$. Human embryonic kidney (HEK) 293 cells harboring the adenovirus E1A region, human lung epithelial cells (BEAS-2B), and human lung carcinoma cell lines A549 and NCI-H1650 were obtained from American Type Culture Collection (ATCC; Rockville, MD, USA) and maintained in Dulbecco's modified Eagle's medium (DMEM; GIBCO-BRL) or Roswell Park Memorial Institute (RPMI) 1640 medium (GIBCO-BRL) containing $5 \% \mathrm{FBS}$ at $37^{\circ} \mathrm{C}$ with $5 \% \mathrm{CO}_{2}$.

\section{CIK induction}

For CIK induction, $2 \times 10^{6}$ cells $/ \mathrm{ml}$ were incubated in IMDM containing $1,000 \mathrm{U} / \mathrm{mL}$ of recombinant human IFN- $\gamma$ (rIFN- $\gamma$, Sigma-Aldrich, St. Louis, MO, USA; SRP3058) for 24 hours, followed by the addition of $400 \mathrm{ng} / \mathrm{mL}$ CD3 monoclonal antibody (mAb; T\&L Biotechnology, Beijing, China, TL-101) and $300 \mathrm{U} / \mathrm{mL}$ recombinant human IL-2 (rIL-2; Sigma-Aldrich; IL002) at $37{ }^{\circ} \mathrm{C}$ with $5 \% \mathrm{CO}_{2}$ for 14 days. Every 3 days, half of the medium was 
replaced with fresh medium containing $300 \mathrm{U} / \mathrm{mL}$ rIL-2. The phenotype of CIK cells was determined, and CD56positive cells were isolated. CIK cells were enriched after expansion by immunomagnetic selection using CD56 microbeads (Miltenyi Biotec, Bergisch-Gladbach, Germany) according to the manufacturer's protocol. Fluorescein-5isothiocyanate (FITC)-human leukocyte antigen class I (HLA-I) antibody (Thermo Fisher Scientific, Waltham, MA, USA) was added to the CIK cells and incubated at $4{ }^{\circ} \mathrm{C}$ in the dark for 30 minutes. The HLA-I' CIK cells were analyzed using a FACSCalibur flow cytometer (BD, Heidelberg, Germany).

\section{Flow cytometry assay}

FACS flow cytometry analysis was performed to detect changes in CIK cell percentages and natural killer (NK) cell receptor profile expressions on CIK cells. Cells were washed twice with HyClone Dulbecco's PBS (1×). Receptor antibodies anti-CD3 and anti-CD56 (Invitrogen, Waltham, MA, USA) were added. We then conducted an assay of the receptors on CIK cells with the FACSCalibur flow cytometer (BD).

\section{Cell viability assay}

The cell viability of BEAS-2B, A549, and NCI-H1650 cells cocultured with CIK cells expressing enhanced green fluorescent protein (EGFP), TRAIL-IETD, MnSOD, and TRAIL-(IETD)-MnSOD was determined using cell counting kit-8 (CCK-8) assay (Beyotime Biotechnology, Shanghai, China) and soft agar clonogenic assay. After 48 hours co-cultivation in 96 -well plates $\left(5 \times 10^{4}\right.$ cells/well $)$, cells were harvested, washed, and incubated with $10 \mu \mathrm{L} /$ well CCK- 8 solution for 60 minutes and then assessed using a Bio-Rad microplate reader (Bio-Rad Laboratories, Inc., Hercules, CA, USA; $450 \mathrm{~nm}$ ). For the soft agar colony formation assay, cells were plated into 6-well plates with a density of 500 cells/well for 14 days, with the medium replaced every 3 days. The cells were then fixed (absolute methanol, 10 minutes) and stained with crystal violet (Beyotime), and colony (>50 cells) numbers were counted using microscopy. The cell viability of NCI-H1650, A549, and BEAS-2B was determined at different effector-totarget (E:T) ratios of 5:1, 10:1, 20:1, respectively, under cocultivation with CIK cells expressing the TRAIL-IETDMnSOD fusion gene. Each treatment was performed in triplicate.

\section{Plasmid and construction of ZD55-TRAIL-IETD- $M n S O D$ recombinant adenovirus}

Adenovirus packaging vectors pBHGE3, pCA13, and pcDNA3.1-TRAIL, and shuttle vectors pShuttle and pShuttle-ZD55 were preserved in our laboratory. All plasmid constructions were performed using restrictive enzyme digestion, polymerase chain reaction (PCR), DNA ligation, DH5 a E. coli cloning, and DNA sequencing. TRAIL-IETD gene fragments were obtained from pcDNA3.1-TRAIL by amplification, and the pCA13TRAIL-IETD was constructed by subcloning into the SalI and XbaI sites of the pCA13 vector. The pShuttle-D55TRAIL-IETD was constructed using pCA13-TRAILIETD and pShuttle-D55 by restrictive enzyme digestion (BgIII), ligation (T4 DNA ligase; Takara Bio Inc., Kusatsu, Shiga, Japan), cloning, and sequencing. The pShuttle-D55EGFP was constructed similarly. The pCA13-MnSOD was constructed by subcloning MnSOD into the EcoRI and $\mathrm{XbaI}$ sites of the pCA13 vector, and the pShuttle-MnSOD was assembled from BglII digestion from pCA13-MnSOD and pShuttle-D55. Accordingly, the pCA13-TRAIL-IETDMnSOD plasmid was assembled from pCA13-TRAIL and pCA13-MnSOD overlap PCR. The construction of pShuttle-D55-TRAIL-IETD-MnSOD was performed by inserting the TRAIL-IETD-MnSOD expression cassette from pCA13-TRAIL-IETD-MnSOD (BgIII digestion) into pZD55.

\section{Generation of recombinant adenovirus, cell infection, and co-cultivation}

The pShuttle-D55-EGFP, pShuttle-D55-TRAIL-IETD, pShuttle-D55-MnSOD, and pShuttle-D55-TRAIL-IETDMnSOD vectors were digested using PmeI and recombined in pAdEasy-1 BJ-5183. PacI digestion and transfection into HEK293 cells was then performed and the recombinant oncolytic adenoviruses ZD55-EGFP, ZD55-TRAIL-IETD, ZD 55-MnSOD, and ZD 55-TRAIL-(IETD)-MnSOD carrying EGFP, TRAIL-IETD, MnSOD, and TRAIL(IETD)-MnSOD were generated, respectively. The identification of recombinant oncolytic adenoviruses was confirmed using PCR.

Recombinant oncolytic adenoviruses were used for infection of CIK cells with a density of $5 \times 10^{5}$ cells/well in 24-well plates for 48 hours. CIK cells stably expressing EGFP, TRAIL-IETD, MnSOD, and TRAIL-(IETD)MnSOD were identified using PCR methods and were 
cocultured with BEAS-2B, A549, and NCI-H1650 cells at $37^{\circ} \mathrm{C}$ with $5 \% \mathrm{CO}_{2}$.

\section{In vitro transwell assay}

Cell invasion ability was determined using transwell assay with Matrigel-coated chambers (Corning Inc., Corning, NY, USA). Cell invasion assay was performed by placing $1 \times 10^{4}$ cells and serum-free medium into the top Matrigelcoated chambers and completed medium (10\% FBS) into the bottom chambers. Cells were incubated for 72 hours at $37{ }^{\circ} \mathrm{C}$ with $5 \% \mathrm{CO}_{2}$. Cells adhered to the upper surfaces of filter membranes were removed, and cells adhered to undersurfaces were fixed, washed, and stained with crystal violet. Digital photographs from 10 randomly selected fields were captured using an Olympus microscope (Olympus Corporation, Tokyo, Japan).

\section{Western blot analysis}

Proteins were extracted from cocultured cells using lysis buffer (Beyotime). After quantification determination, protein samples were analyzed following standard methods. Proteins were separated by $10 \%$ sodium dodecyl sulphatepolyacrylamide gel electrophoresis (SDS-PAGE) and electrotransferred onto polyvinylidene difluoride (PVDF) membranes (Millipore Corp., Billerica, MA, USA). Antibodies against TRAIL protein (1:1,000, Santa Cruz Biotechnology, Santa Cruz, CA, USA), MnSOD protein (1:500, Santa Cruz), and glyceraldehyde 3-phosphate dehydrogenase (GAPDH) protein (1:1,000, Sigma-Aldrich) were used as specific primary antibodies for primary incubation. Horseradish peroxidase (HRP) goat anti-rabbit/ rat immunoglobulin $\mathrm{G}$ ( $\mathrm{IgG}$ ) secondary antibodies (1: 20,000, Boster Biology Technology, Ltd., Wuhan, China) were used for secondary incubation. Digital analysis was implemented using Image-Pro Plus 6.0 software (Media Cybernetics Inc., Bethesda, MD, USA). GAPDH (1:20,000, Boster) was used as the reference protein.

\section{Enzyme-linked immunosorbent assay (ELISA) measurement}

IFN- $\gamma, \mathrm{TNF}-\alpha$, and lactate dehydrogenase (LDH) contents in cellular cultures were detected using corresponding commercial kits (Elabscience Biotechnology, Wuhan, China). After co-cultivation for 48 hours, the cell culture supernatants were collected by centrifugation, and the levels of IFN- $\gamma, \mathrm{TNF}-\alpha$, and LDH were detected following the protocols from the kits (E-EL-H0108, E-EL-H0109, and E-EL-H0556). Three replicate wells were set for each sample, and each experiment was performed 3 times.

\section{Animal experiments}

Seventy nude mice (5 weeks old, 16-21 g) were purchased from Shanghai Laboratory Animals Center (SLAC, Shanghai, China) and adaptively fed for 1 week in the Shenzhen Hospital of Southern Medical University animal center. Each mouse then received a subcutaneous injection of $1 \times 10^{7}$ A549 cells into the lower right flank. When tumor size reached $1 \times 1 \times 1 \mathrm{~cm}^{3}$, the mice were randomly divided into six groups and given an intravenous tail injection with CIK cells expressing EGFP, TRAIL-IETD, MnSOD, a254nd TRAIL(IETD)-MnSOD $\left(4 \times 10^{7} / 0.2 \mathrm{~mL}\right)$ every 3 days for a total of 9 times $(0,3,6,9,12,15,18,21$, and 24 days). A blank control group received nothing, and a negative control group received CIK cells expressing EGFP. Tumor dispersion was calculated as $(\mathrm{a} \times \mathrm{b} \times \mathrm{b}) / 2$ where $\mathrm{a}$ is the largest diameter and $\mathrm{b}$ is the perpendicular diameter. Tumors were separated and volume was recorded. Blood and tumor samples were collected and prepared for further analyses. All animal experiments were performed according to the Guide for the Care and Use of Laboratory Animals (20). The animal protocol was maintained in accordance with the guidelines of the animal care committee of the Shenzhen Hospital of Southern Medical University for the care and use of animals, and all procedures were approved by the ethics committee of the Shenzhen Hospital of Southern Medical University.

\section{Histopathology and immunohistochemistry (IHC)}

Harvested tumor samples were fixed, paraffin embedded, and sectioned into $4-\mu \mathrm{m}$ sections onto slides. For IHC analysis, sections were stained with Ki-67 antibody (1:500; CST Biological Reagents Co., Ltd., Shanghai, China) at $4{ }^{\circ} \mathrm{C}$ overnight, followed by incubation in avidinbiotin-peroxidase complex reagent (Vector Laboratories, Burlingame, CA, USA). For histopathology analysis, sections were stained with hematoxylin and eosin (HE) (Beyotime). Images were captured using a BX51 microscope (Olympus).

\section{Measurement of serum IFN- $\gamma, T N F-\alpha$, and $L D H$ levels}

Blood samples were collected for the measurement of serum 
IFN- $\gamma$, TNF- $\alpha$, and LDH levels using the aforementioned methods.

\section{Statistical analysis}

All data reported in this study are expressed as mean \pm standard deviation. Differences were analyzed using Student's $t$-test. GraphPad Prism 6.0 software was used for statistical analyses. Difference was considered significant and very significant when ${ }^{*} \mathrm{P}<0.05$ and ${ }^{* *} \mathrm{P}<0.05$, respectively.

\section{Results}

\section{In vitro proliferation inbibition of ZD55-TRAIL-IETD- MnSOD in lung cancer cells}

CIK cells were obtained from peripheral blood mononuclear cells of lung cancer patients using IFN- $\gamma$, IL2 , and $\mathrm{CD} 3$ monoclonal antibodies. The $\mathrm{CD} 3{ }^{+} \mathrm{CD} 56^{+} \mathrm{CIK}$ cell population was detected by FACS flow cytometry, which indicated that the cells had gradually increased over 21 days (Figure S1A). CIK cells stably expressing exogenous genes were established and no significant difference regarding in vitro cytotoxicity was found among CIK cells transduced with oncolytic adenoviruses ZD55-EGFP, ZD55-TRAILIETD, ZD55-MnSOD, ZD55-TRAIL + ZD55-MnSOD, and ZD55-TRAIL-IETD-MnSOD (Figure S1B). To investigate the cytopathic effects of ZD55-TRAIL-IETDMnSOD, we cocultured CIK cells stably expressing exogenous genes with lung cancer cell lines NCI-H1650 and A549 for 48 hours. Human lung epithelial cells BEAS2B were used as normal control cells. CCK8 assay showed that the cell viability of NCI-H1650 and A549 cells was significantly reduced after coculturing with CIK cells expressing TRAIL + MnSOD and TRAIL-IETD-MnSOD (Figure 1A). In A549 cells, the cell viability was significantly reduced by coculturing with CIK cells expressing TRAIL, MnSOD, TRAIL + MnSOD, and TRAIL-IETD-MnSOD (Figure $1 A$ ). The cell viability of BEAS-2B cells was not affected by TRAIL-IETD-MnSOD.

We observed that compared with the blank group, the colony formation ability of NCI-H1650 and A549 cells was most significantly decreased by CIK cells expressing TRAIL-IETD-MnSOD, followed in order by TRAIL + MnSOD, MnSOD, TRAIL, and EGFP (Figure 1B,1C). CIK cells expressing the TRAIL-IETD-MnSOD fusion gene obviously decreased colony numbers of NCI-H1650 and A549 cells compared with CIK cells expressing both genes
(TRAIL and MnSOD, Figure 1B). In addition, we found the colony formation ability of BEAS-2B cells was decreased by CIK cells expressing ZD55-TRAIL and ZD55-TRAILIETD-MnSOD compared with the blank control group and cells expressing the MnSOD gene. We further demonstrated the cytopathic effects of ZD55-TRAIL-IETD-MnSOD in both HLA-I ${ }^{+}$and HLA-I ${ }^{-}$of human lung carcinoma cell lines A549 and NCI-H1650 as well as at different E:T ratios in cocultivated cells (Figure S1C,S1D).

These results suggested that co-cultivation with CIK cells expressing the TRAIL-IETD-MnSOD fusion gene as well as single or combined TRAIL and MnSOD genes were inhibitory to the proliferation of lung cancer cells NCI-H1650 and A549. However, the TRAIL-IETDMnSOD fusion gene showed a greater inhibitory effect on cell proliferation than combined TRAIL and MnSOD genes. A mild proliferation inhibitory effect was seen in BEAS-2B cells.

\section{ZD55-TRAIL-IETD-MnSOD fusion gene suppresses NCI-H1650 and A549 cell invasion}

In vitro transwell invasion assay showed that co-cultivation with CIK cells expressing the TRAIL-IETD-MnSOD fusion gene, and single or combined TRAIL and MnSOD genes significantly reduced the invasion ability of cancer cells (Figure $2 A, 2 B$ ). The number of invaded NCI-H1650 and A549 cells were decreased by (in order) ZD55-TRAILIETD-MnSOD > ZD55-TRAIL + ZD55-MnSOD > ZD55-MnSOD > ZD55-TRAIL > ZD55-EGFP. In BEAS$2 \mathrm{~B}$ cells, invaded cell numbers were significantly decreased by co-cultivation with CIK cells harboring oncolytic adenoviruses ZD55-TRAIL-IETD-MnSOD and ZD55TRAIL but not combined ZD55-TRAIL and ZD55MnSOD or ZD55-MnSOD alone. These results suggest that CIK cells carrying oncolytic adenovirus ZD55-TRAILIETD-MnSOD had the highest cytopathy effectiveness for cancer cells. CIK cells harboring oncolytic adenoviruses ZD55-TRAIL and ZD55-MnSOD might be the most reliable strategy yet because of the cytopathic effect of ZD55-TRAIL-IETD-MnSOD on BEAS-2B cells.

\section{ZD55-TRAIL-IETD-MnSOD increased lung cancer cell cytokines}

Figure 3 shows that the content of cellular IFN- $\gamma$ and TNF- $\alpha$ cytokines in A549 and NCI-H1650 cells was dramatically increased by CIK cells carrying oncolytic 



Blank
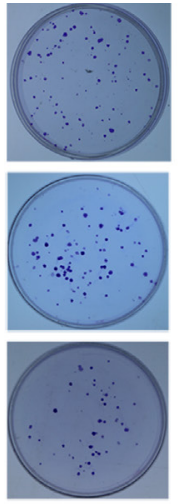

ZD55
A549



A549
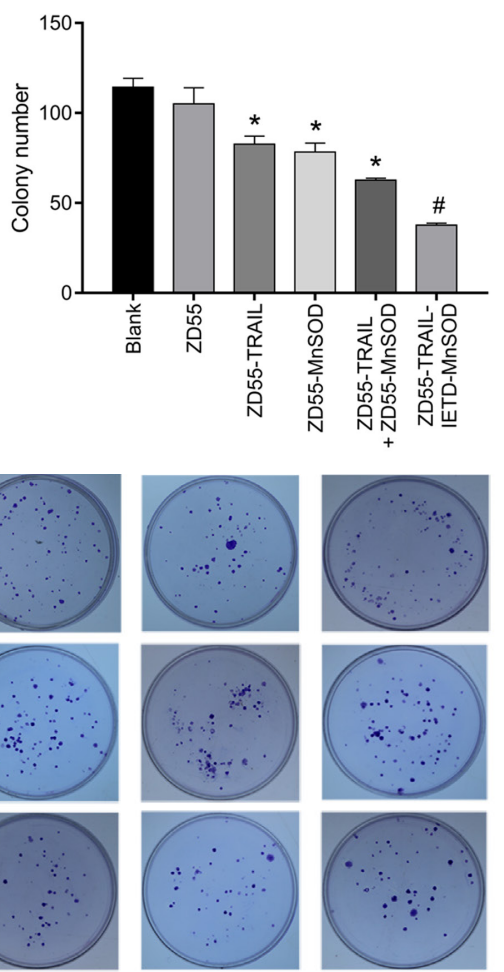

ZD55-TRAIL
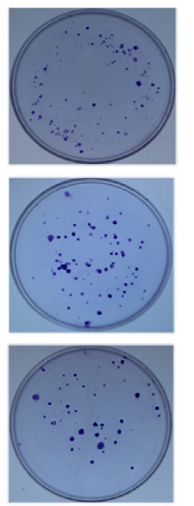

ZD55-MnSOD

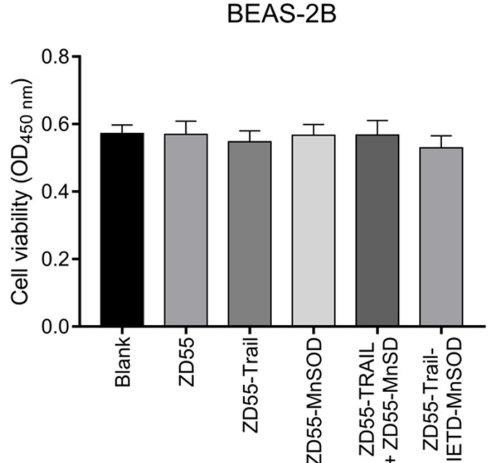

BEAS-2B
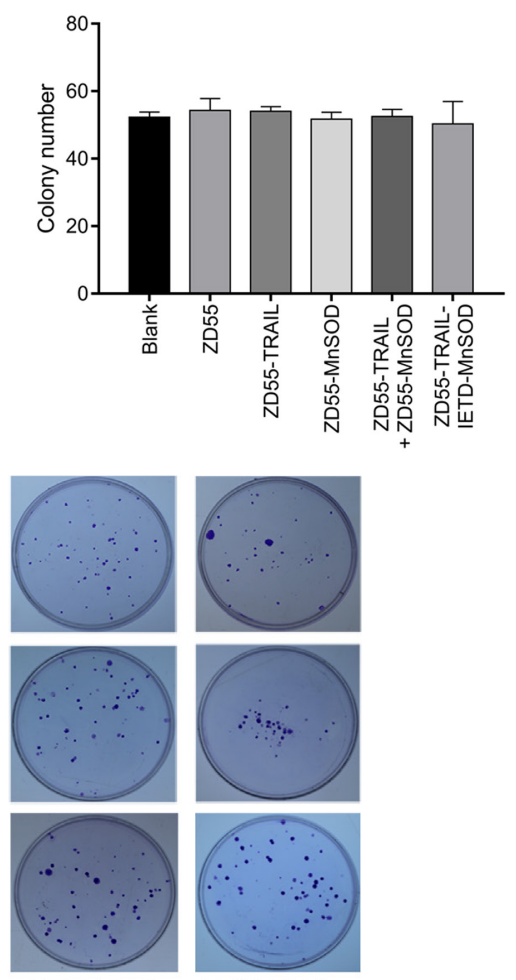

ZD55-TRAIL + ZD55-MnSOD IETD-MnSOD

Figure 1 The inhibitory effect of oncolytic adenovirus ZD55-TRAIL-IETD-MnSOD on cancer cell proliferation. (A) Cell viability by CCK-8 analysis at 48 hours post co-cultivation; (B) colony formation assay of different cells at 14 days post co-cultivation with CIK cells harboring oncolytic adenoviruses ZD55-EGFP, ZD55-TRAIL, ZD55-MnSOD, ZD55-TRAIL + ZD55-MnSOD, and ZD55-TRAILIETD-MnSOD; (C) representative images of the soft agar colony formation assay (stain method: crystal violet; magnification: $\times 40$ ). The blank group received CIK alone. * $\mathrm{P}<0.05 ;{ }^{*}, \mathrm{P}<0.05$. Differences were compared with the blank control or others indicated by lines.

adenoviruses (in order) ZD55-TRAIL-IETD-MnSOD > ZD55-TRAIL+ZD55-MnSOD > ZD55-MnSOD > ZD55TRAIL > ZD55-EGFP (Figure 3A,3B). No alteration was seen in the content of IFN- $\gamma$ and TNF- $\alpha$ cytokines in BEAS-2B cells cocultured with CIK cells. These results might explain the increased inflammation in oncolytic adenoviruses-treated lung cancer cells.

We determined changes in $\mathrm{LDH}$ content to investigate the injury of recombinant oncolytic adenoviruses to cancer cell membranes. Figure $3 C$ shows the LDH level was significantly elevated by CIK cells harboring oncolytic adenoviruses, in the same order as IFN- $\gamma$ and TNF- $\alpha$. No 




A549

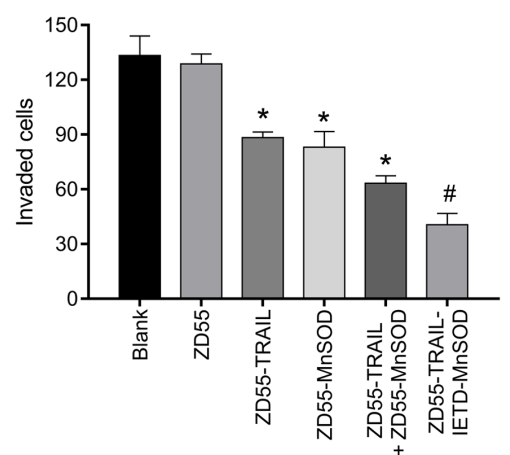

BEAS-2B

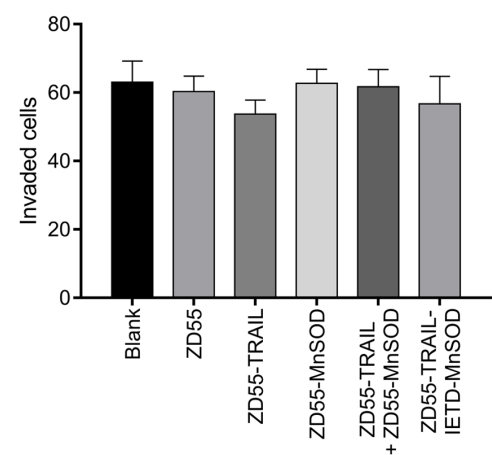



Figure 2 ZD55-TRAIL-IETD-MnSOD inhibits lung cancer cell invasion. (A) Digital analysis of invaded cell numbers; (B) representative images of transwell assay. Three cell lines were cocultured with PBMC-derived CIK cells harboring oncolytic adenoviruses ZD55-EGFP, ZD55-TRAIL, ZD55-MnSOD, ZD55-TRAIL + ZD55-MnSOD, and ZD55-TRAIL-IETD-MnSOD (stain method: crystal violet; magnification: $\times 200) .^{*}, \mathrm{P}<0.05 ;{ }^{\#}, \mathrm{P}<0.05$.

increment was seen in LDH content in BEAS-2B cells. Accordingly, we demonstrated that the cell membranes had been obviously damaged by ZD55-TRAIL-IETD-MnSOD, followed in order by ZD55-TRAIL + ZD55-MnSOD, ZD55-MnSOD, ZD55-TRAIL, and ZD55-EGFP.

\section{In vivo antitumoral efficacy of ZD55-TRAIL-IETD- MnSOD}

The in vivo antitumoral efficacy of ZD55-TRAIL-IETDMnSOD was determined using a subcutaneous tumor model by injecting A549 cells. A xenograft model antitumor assay was performed by intravenous tail injection of CIK cells harboring oncolytic adenoviruses ZD55-TRAILIETD, ZD 55 -MnSOD, and ZD55-TRAIL-(IETD)MnSOD. We found ZD55-TRAIL-IETD-MnSOD showed a stronger antitumor effect than the others $(\mathrm{P}<0.01$, Figure $4 A$ ). Histopathology analysis using $\mathrm{HE}$ staining showed ZD55-TRAIL-IETD-MnSOD induced the most significant cytopathic effects on tumors (Figure 4B). IHC staining showed that ZD55-TRAIL-IETD-MnSOD most significantly decreased $\mathrm{Ki}-67$ positive cells in tumor tissues (Figure 4C). These results suggested the in vivo antitumoral efficacy of CIK cells harboring oncolytic adenovirus ZD55TRAIL-IETD-MnSOD.

\section{ZD55-TRAIL-IETD-MnSOD increases levels of IFN- $\gamma$, $T N F-\alpha$, and $L D H$}

Our data showed that IFN- $\gamma$, TNF- $\alpha$, and LDH were significantly elevated by ZD55-TRAIL-IETD-MnSOD, followed in order by ZD55-TRAIL + ZD55-MnSOD, ZD55-MnSOD, ZD55-TRAIL, and ZD55-EGFP. The contents of IFN- $\gamma$, TNF- $\alpha$, and LDH in mice administrated with CIK cells harboring oncolytic adenovirus ZD55TRAIL-IETD-MnSOD were significantly higher than the other treatments (Figure $5 A-5 C, \mathrm{P}<0.01$ ), suggesting an increased inflammatory status in mice. In addition, the 

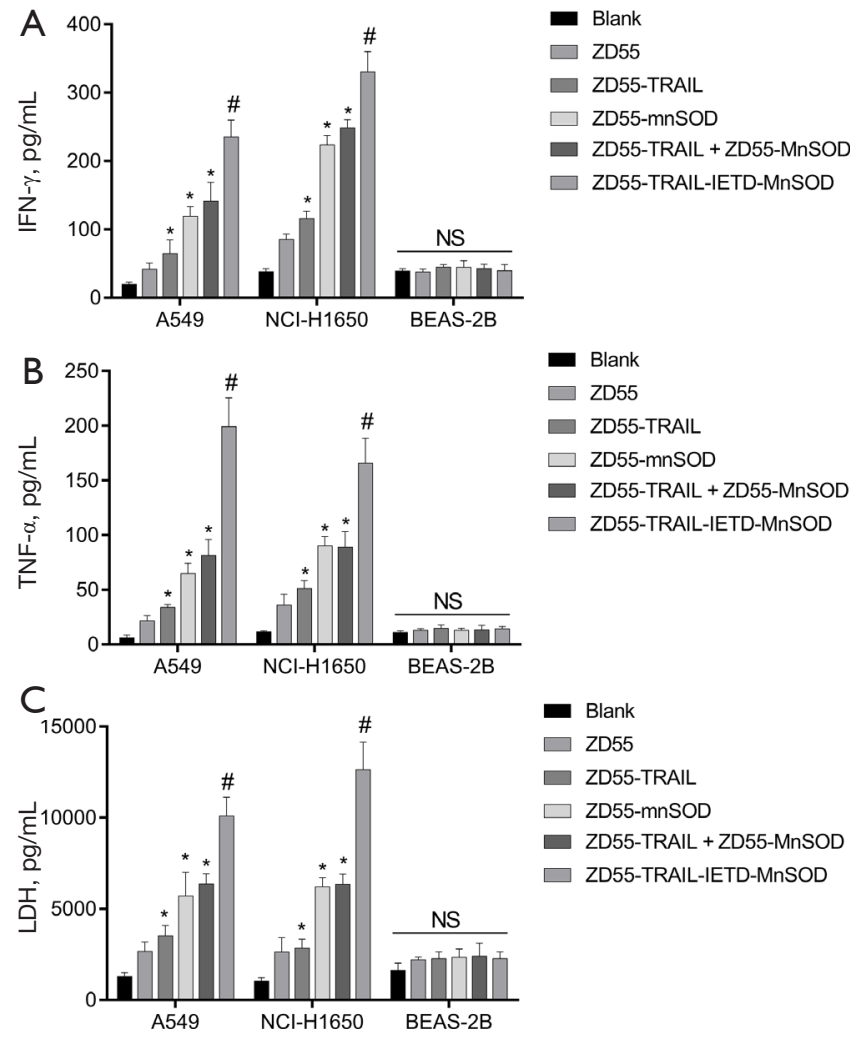

Figure 3 Effect of ZD55-TRAIL-IETD-MnSOD on cytokines and cell damage. The contents of IFN- $\gamma(\mathrm{A}), \mathrm{TNF}-\alpha(\mathrm{B})$, and $\mathrm{LDH}(\mathrm{C})$ in cellular cultures were detected using ELISA kits. Cells were cocultured with CIK cells harboring oncolytic adenoviruses ZD55-EGFP, ZD55-TRAIL, ZD55-MnSOD, ZD55-TRAIL + ZD55-MnSOD, and ZD55-TRAIL-IETD-MnSOD for 48 hours. * $\mathrm{P}<0.05$;, $\mathrm{P}<0.05$; NS, no significance. $\mathrm{LDH}$, lactate dehydrogenase.

expressions of TRAIL and MnSOD in tumor tissues were elevated as the levels of IFN- $\gamma, \mathrm{TNF}-\alpha$, and $\mathrm{LDH}$ increased (Figure 5D), validating the inducing role of ZD55-TRAILIETD-MnSOD on inflammatory status.

\section{Discussion}

CIK cell immunotherapy strategy depends on enhanced immune responses to cancers (12-14), and CTGVT confers cancer cells with enhanced apoptosis and cytopathy $(6,10)$. Previous study has found that OV-mediated TRAIL or MnSOD could significantly inhibit human cancer $(21,22)$. However, combined therapy of OV-mediated TRAIL or MnSOD in lung cancer through CIK is still unknown. The synergic effect of CIK cell immunotherapy and CTGVT harbors higher efficacy against human tumors $(18,19)$. Our study suggested that combined administration of CIK cells and oncolytic adenovirus ZD55 harboring TRAIL-IETDMnSOD showed high efficacy against lung cancer cell proliferation and invasion, showing the cytopathic effects in vitro in A549 and NCI-H1650 cells and antitumor effects in vivo in an A549 xenograft mouse model.

TRAIL is an apoptosis-inducing gene (6) and is preferentially cytotoxic towards cancer cells and nontoxic to normal cells (23), just as oncolytic adenovirus is preferentially replicated in cancer cells but is restricted in normal cells $(8,9)$. TRAIL-mediated apoptosis is associated with various factors $(24,25)$. It has been reported that TRAIL-armed oncolytic adenovirus ZD55 induces apoptosis in human hepatocellular carcinoma cells $(7,9,26)$, pancreatic cancer cells (27), and lung cancer cells (5). Yang et al. found that oncolytic adenovirus ZD55-TRAIL showed cytotoxicity against A549 sphere cells and prolonged survival status of A549 xenograft mice (5). In addition, MnSOD is a mitochondria-resident enzyme that detoxifies superoxide harboring bilateral activity in tumors $(28,29)$. The overexpression of MnSOD has been reported to promote tumor growth and stem-like cell phenotypes (28,30,31), whereas oncolytic adenovirus ZD55-MnSOD has been reported to have an antitumor effect against hepatocellular carcinoma cells (21) and lung cancer cells (8). Consistent with these findings, our results showed that the oncolytic adenovirus ZD55 harboring TRAIL or MnSOD alone and the TRAIL-MnSOD infusion gene had a cytopathic and antitumor effect against lung cancer cells. Single treatment with oncolytic adenovirus ZD55-TRAIL or ZD55-MnSOD gene, or in combination, obviously decreased A549 and NCI-H1650 cell proliferation, colony formation, invasion, and tumor growth. Further, ZD55-MnSOD displayed stronger cytopathic and antitumor effects than ZD55TRAIL, while ZD55-TRAIL-IETD-MnSOD had the highest effect. These results were consistent with those from a study by Zhang et al. (8), suggesting the synergic effect of oncolytic adenovirus ZD55 harboring both TRAIL and MnSOD genes against lung cancer.

Our antitumor experiments with oncolytic adenoviruses were all performed using CIK cell delivery. Oncolytic adenoviruses ZD55 harboring TRAIL, MnSOD, and TRAIL-IETD-MnSOD were transduced into CIK cells, which were then cocultured in vitro with lung cancer cells (A549 and NCI-H1650) or normal human lung epithelial cells (BEAS-2B), or injected in vivo into an A549 xenograft model. It has been found that TRAIL causes apoptosis 
A
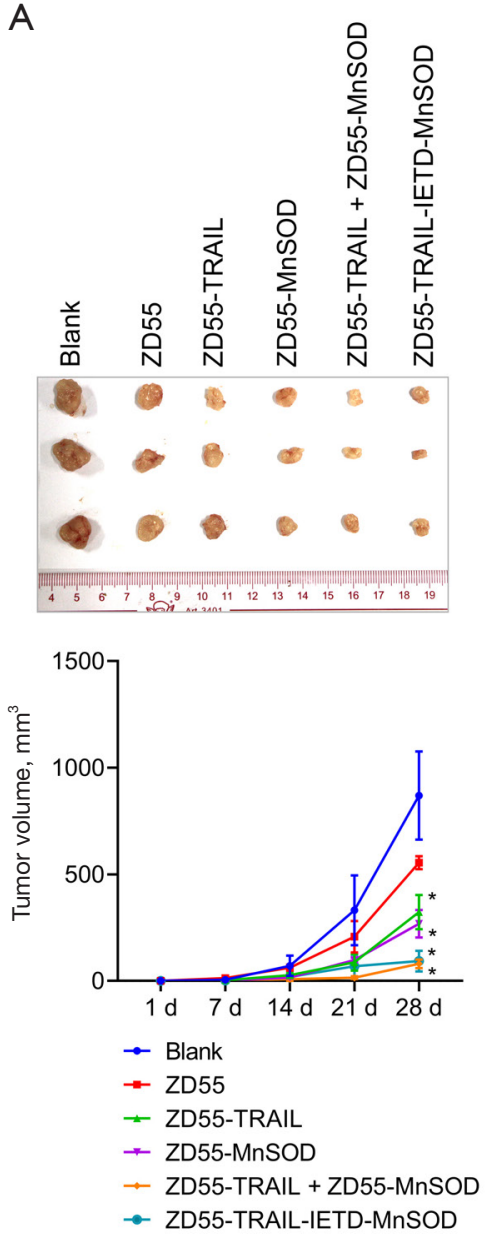

B

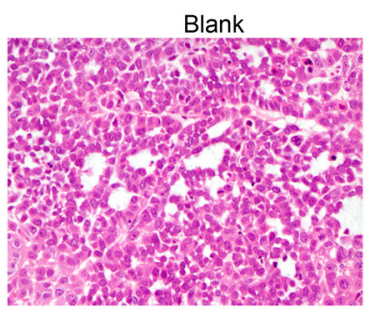
ZD55-MnSOD

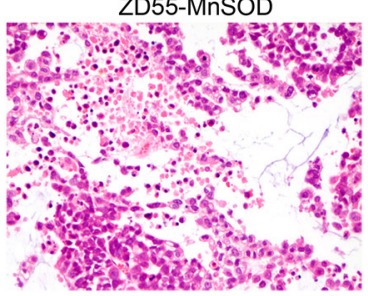

C

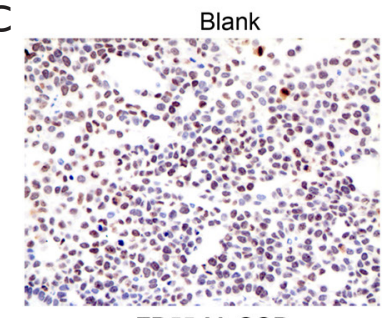

ZD55-MnSOD

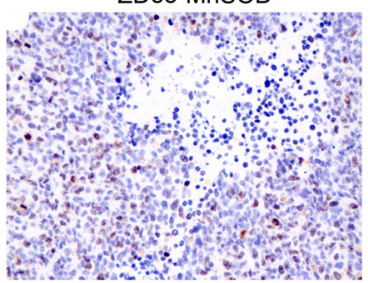



ZD55-TRAIL + ZD55-MnSOD ZD55-TRAIL-IETD-MnSOD


ZD55

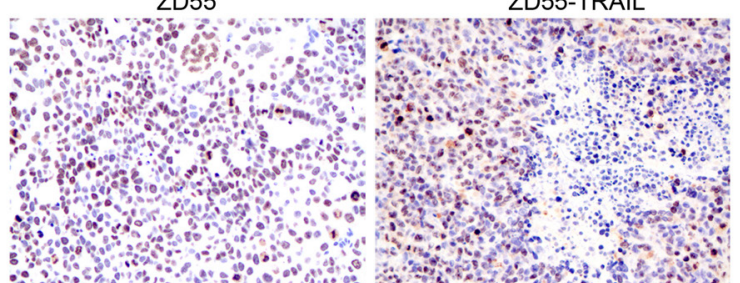

ZD55-TRAIL + ZD55-MnSOD ZD55-TRAIL-IETD-MnSOD

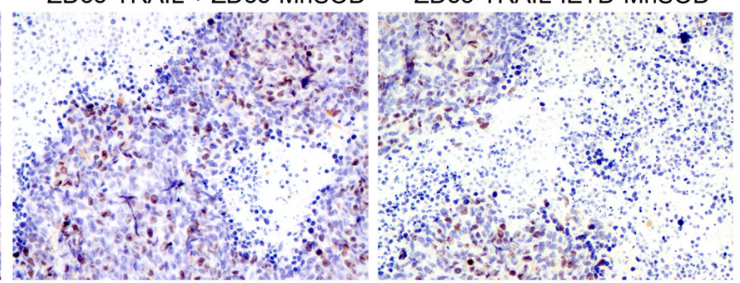

Figure 4 In vivo antitumoral efficacy of ZD55-TRAIL-IETD-MnSOD in a mouse model. An A549 xenograft model underwent tail intravenous injection with CIK cells harboring oncolytic adenoviruses twice per week for 10 weeks. (A) Tumor sizes in the A549 xenograft mouse model; (B,C) the representative images of the histopathology by using H\&E staining and immunohistochemistry (Ki-67) using DAB developing (magnification: $\times 200$ ). ${ }^{*}, \mathrm{P}<0.05$.

of various tumors but leaves normal cells unharmed, constituting a physiologic pathway of CD4 T cell-mediated cytotoxicity against tumor cells (32). However, TRAIL can also induce apoptosis of activated human T cells (33), neutrophils (34), and hepatocytes (35). Considering the potential killing effect of TRAIL on CIK expressing TRAIL, we determined the in vitro cytotoxicity of CIK cells expressing TRAIL and found that there was no significant difference on cell viability among CIK cells transduced with oncolytic adenoviruses ZD55-EGFP, ZD55-TRAILIETD, ZD55-MnSOD, ZD55-TRAIL + ZD55-MnSOD, and ZD55-TRAIL-IETD-MnSOD, indicating the overexpression of TRAIL did not affect CIK growth. It has been reported that oncolytic adenoviruses lack tumor specificity in lung cancer cells (8). Our study revealed that oncolytic adenoviruses administration inhibited cancer cell growth and invasion, and they also selectively inhibited the proliferation and invasion ability of BEAS-2B cells. The proliferation, colony formation, and invasion abilities of BEAS-2B cells were inhibited by oncolytic adenoviruses ZD55 carrying TRAIL and TRAIL-IETD-MnSOD. CIK cell antitumor cytotoxicity has been found to be HLA-independent (20). CIK cells produce inflammatory cytokines, including IFN- $\gamma$, IL-2, and TNF- $\alpha$, alleviate cytotoxicity-induced immune damage, and improve the inflammatory status in cancer patients with drugs or 

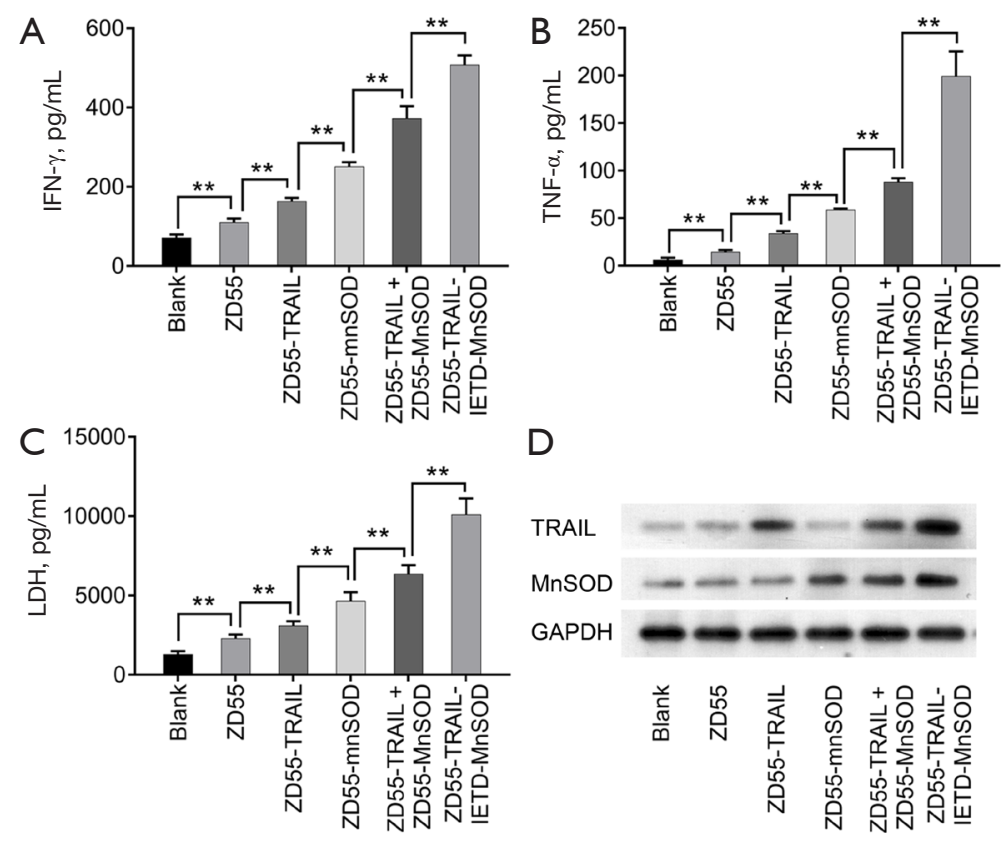

$\mathrm{D}$

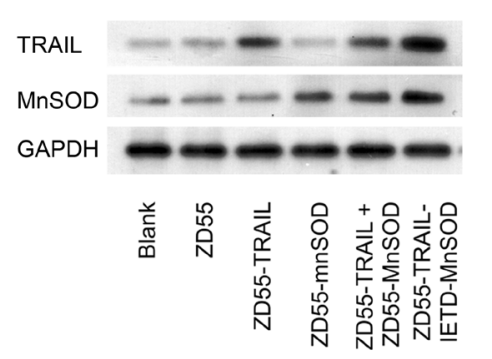

Figure 5 Levels of IFN- $\gamma$, TNF- $\alpha$, and LDH in a mouse model. IFN- $\gamma$ (A), TNF- $\alpha$ (B) and LDH (C) serum contents were detected using commercial ELISA kits. (D) The expressions of TRAIL and MnSOD in tumor tissues were detected by western blotting. **, P<0.01. LDH, lactate dehydrogenase.

chemoradiotherapy (13). The production of LDH, IFN- $\gamma$, and TNF- $\alpha$ in A549 and NCI-H1650 cells and the A549 xenograft model were significantly increased by CIK celldelivered oncolytic adenoviruses, revealing an improved inflammatory status. However, the contents of LDH, IFN- $\gamma$, and TNF- $\alpha$ in BEAS-2B cellular culture were not influenced by oncolytic adenoviruses. These results suggest that the CIK cell-delivered oncolytic adenovirus may be relatively viable for lung cancer therapy.

In conclusion, our study transduced oncolytic adenoviruses ZD55 harboring TRAIL, MnSOD, and TRAIL-IETD-MnSOD into A549 and NCI-H1650 cells via CIK cells delivery. In vitro experiments showed the cytopathic effects of CIK cells carrying ZD55-TRAIL, ZD 55-MnSOD, and ZD 55-TRAIL-IETD-MnSOD against A549 and NCI-H1650 cell proliferation, colony formation, and invasion, and the anti-tumor effect of TRAIL and MnSOD connected with IETD was more effective compared with ZD55-TRAIL or ZD55-MnSOD. In vivo experiments demonstrated the antitumor effects of the combination of CIK cells and oncolytic adenoviruses. In addition, both in vitro and in vivo experiments revealed the synergistic effect of ZD55-TRAIL and ZD55-MnSOD on lung cancer.

\section{Acknowledgments}

Funding: This research was funded by the Miao Miao breeding plan project of Shenzhen Hospital of Southern Medical University (No. 2016MM08).

\section{Footnote}

Reporting Checklist: The authors have completed the ARRIVE reporting checklist. Available at https://dx.doi. org/10.21037/atm-21-4479

Data Sharing Statement: Available at https://dx.doi. org/10.21037/atm-21-4479

Conflicts of Interest: All authors have completed the ICMJE uniform disclosure form (available at https://dx.doi. org/10.21037/atm-21-4479). The authors have no conflicts of interest to declare.

Ethical Statement: The authors are accountable for all aspects of the work in ensuring that questions related to the accuracy or integrity of any part of the work are appropriately investigated and resolved. The animal 
protocol was maintained in accordance with the guidelines of the animal care committee of the Shenzhen Hospital of Southern Medical University for the care and use of animals, and all procedures were approved by the ethics committee of the Shenzhen Hospital of Southern Medical University.

Open Access Statement: This is an Open Access article distributed in accordance with the Creative Commons Attribution-NonCommercial-NoDerivs 4.0 International License (CC BY-NC-ND 4.0), which permits the noncommercial replication and distribution of the article with the strict proviso that no changes or edits are made and the original work is properly cited (including links to both the formal publication through the relevant DOI and the license). See: https://creativecommons.org/licenses/by-nc-nd/4.0/.

\section{References}

1. Yang HX, Woo KM, Sima CS, et al. Long-term Survival Based on the Surgical Approach to Lobectomy For Clinical Stage I Nonsmall Cell Lung Cancer: Comparison of Robotic, Video-assisted Thoracic Surgery, and Thoracotomy Lobectomy. Ann Surg 2017;265:431-7.

2. White A, Joseph D, Rim SH, et al. Colon cancer survival in the United States by race and stage (2001-2009): Findings from the CONCORD-2 study. Cancer 2017;123 Suppl 24:5014-36.

3. Paz-Ares L, Tan EH, O'Byrne K, et al. Afatinib versus gefitinib in patients with EGFR mutation-positive advanced non-small-cell lung cancer: overall survival data from the phase IIb LUX-Lung 7 trial. Ann Oncol 2017;28:270-7.

4. Solomon BJ, Kim DW, Wu YL, et al. Final Overall Survival Analysis From a Study Comparing First-Line Crizotinib Versus Chemotherapy in ALK-MutationPositive Non-Small-Cell Lung Cancer. J Clin Oncol 2018;36:2251-8.

5. Yang $\mathrm{Y}, \mathrm{Xu} \mathrm{H}$, Huang $\mathrm{W}$, et al. Targeting lung cancer stem-like cells with TRAIL gene armed oncolytic adenovirus. J Cell Mol Med 2015;19:915-23.

6. Luo Q, Song H, Deng X, et al. A Triple-Regulated Oncolytic Adenovirus Carrying MicroRNA-143 Exhibits Potent Antitumor Efficacy in Colorectal Cancer. Mol Ther Oncolytics 2020;16:219-29.

7. Wang SB, Tan Y, Lei W, et al. Complete eradication of xenograft hepatoma by oncolytic adenovirus ZD55 harboring TRAIL-IETD-Smac gene with broad antitumor effect. Hum Gene Ther 2012;23:992-1002.

8. Zhang R, Zhang X, Ma B, et al. Enhanced antitumor effect of combining TRAIL and MnSOD mediated by CEAcontrolled oncolytic adenovirus in lung cancer. Cancer Gene Ther 2016;23:168-77.

9. Galal El-Shemi A, Mohammed Ashshi A, Oh E, et al. Efficacy of combining ING4 and TRAIL genes in cancer-targeting gene virotherapy strategy: first evidence in preclinical hepatocellular carcinoma. Gene Ther 2018;25:54-65.

10. Oberley LW. Mechanism of the tumor suppressive effect of MnSOD overexpression. Biomed Pharmacother 2005;59:143-8.

11. Li XM, Yang YW, Maimaitiyiming XK, et al. Effect of Second mitochondria-derived activator of caspase in combination with Docetaxel on lung cancer cell A549. Int J Clin Exp Med 2015;8:7613-9.

12. Oelsner S, Wagner J, Friede ME, et al. Chimeric antigen receptor-engineered cytokine-induced killer cells overcome treatment resistance of pre-B-cell acute lymphoblastic leukemia and enhance survival. Int J Cancer 2016;139:1799-809.

13. Pan K, Guan XX, Li YQ, et al. Clinical activity of adjuvant cytokine-induced killer cell immunotherapy in patients with post-mastectomy triple-negative breast cancer. Clin Cancer Res 2014;20:3003-11.

14. Wang D, Zhang B, Gao H, et al. Clinical research of genetically modified dendritic cells in combination with cytokine-induced killer cell treatment in advanced renal cancer. BMC Cancer 2014;14:251.

15. Li YC, Zhao L, Wu JP, et al. Cytokine-induced killer cell infusion combined with conventional treatments produced better prognosis for hepatocellular carcinoma patients with barcelona clinic liver cancer B or earlier stage: A systematic review and meta-analysis. Cytotherapy 2016;18:1525-31.

16. Cappuzzello E, Sommaggio R, Zanovello P, et al. Cytokines for the induction of antitumor effectors: The paradigm of Cytokine-Induced Killer (CIK) cells. Cytokine Growth Factor Rev 2017;36:99-105.

17. Zhang J, Zhu L, Du H, et al. Autologous cytokine-induced killer cell therapy in lung cancer patients: a retrospective study. Biomed Pharmacother 2015;70:248-52.

18. Ye JF, Lin YQ, Yu XH, et al. Immunotherapeutic effects of cytokine-induced killer cells combined with CCL21/IL15 armed oncolytic adenovirus in TERT-positive tumor cells. Int Immunopharmacol 2016;38:460-7.

19. Yan $\mathrm{Y}, \mathrm{Xu} \mathrm{Y}, \mathrm{Zhao} \mathrm{Y}$, et al. Combination of E2F-1 promoter-regulated oncolytic adenovirus and cytokine- 
Page 12 of 12

induced killer cells enhances the antitumor effects in an orthotopic rectal cancer model. Tumour Biol 2014;35:1113-22.

20. Research NRCIfLA. Guide for the Care and Use of Laboratory Animals. Astronomy and Astrophysics 2004;327:963-5.

21. Huang F, Ma B, Wang Y, et al. Targeting gene-virusmediated manganese superoxide dismutase effectively suppresses tumor growth in hepatocellular carcinoma in vitro and in vivo. Cancer Biother Radiopharm 2014;29:403-11.

22. Ma H, Liu Y, Liu S, et al. Recombinant adeno-associated virus-mediated TRAIL gene therapy suppresses liver metastatic tumors. Int J Cancer 2005;116:314-21.

23. Hung CM, Liu LC, Ho CT, et al. Pterostilbene Enhances TRAIL-Induced Apoptosis through the Induction of Death Receptors and Downregulation of Cell Survival Proteins in TRAIL-Resistance Triple Negative Breast Cancer Cells. J Agric Food Chem 2017;65:11179-91.

24. Nazim UM, Rasheduzzaman M, Lee YJ, et al. Enhancement of TRAIL-induced apoptosis by 5 -fluorouracil requires activating Bax and p53 pathways in TRAIL-resistant lung cancers. Oncotarget 2017;8:18095-105.

25. Iurlaro R, Püschel F, León-Annicchiarico CL, et al. Glucose Deprivation Induces ATF4-Mediated Apoptosis through TRAIL Death Receptors. Mol Cell Biol 2017;37:e00479-16.

26. Zou H, Zheng YF, Ge W, et al. Synergistic Anti-tumour Effects of Quercetin and Oncolytic Adenovirus expressing TRAIL in Human Hepatocellular Carcinoma. Sci Rep 2018;8:2182.

27. Ge Y, Lei W, Ma Y, et al. Synergistic antitumor effects

Cite this article as: Jiang $\mathrm{R}$, Zhang $\mathrm{Z}$, Liao $\mathrm{X}$, Huang L, Liao Y, Deng W. Combination of oncolytic adenovirus ZD55 harboring TRAIL-IETD-MnSOD and cytokine-induced killer cells against lung cancer. Ann Transl Med 2021;9(20):1527. doi: 10.21037/atm-21-4479
Jiang et al. ZD55-TRAIL-MnSOD and CIK cells in lung cancer

of CDK inhibitor SNS-032 and an oncolytic adenovirus co-expressing TRAIL and Smac in pancreatic cancer. Mol Med Rep 2017;15:3521-8.

28. Hart PC, Mao M, de Abreu AL, et al. MnSOD upregulation sustains the Warburg effect via mitochondrial ROS and AMPK-dependent signalling in cancer. Nat Commun 2015;6:6053.

29. Ekoue DN, Bera S, Ansong E, et al. Abstract 225: Allelic variations in MnSOD and GPx-1 affect metabolism, mitochondrial membrane potential and expression of signaling proteins. Cancer Res 2016;76:abstr 225.

30. Miar A, Hevia D, Muñoz-Cimadevilla H, et al. Manganese superoxide dismutase (SOD2/MnSOD)/catalase and SOD2/GPx1 ratios as biomarkers for tumor progression and metastasis in prostate, colon, and lung cancer. Free Radic Biol Med 2015;85:45-55.

31. Fu Z, Cao X, Yang Y, et al. Upregulation of FoxM1 by MnSOD Overexpression Contributes to Cancer Stem-Like Cell Characteristics in the Lung Cancer H460 Cell Line. Technol Cancer Res Treat 2018;17:1533033818789635.

32. Wang S, El-Deiry WS. TRAIL and apoptosis induction by TNF-family death receptors. Oncogene 2003;22:8628-33.

33. Janssen EM, Droin NM, Lemmens EE, et al. CD4+ T-cell help controls CD8+ T-cell memory via TRAIL-mediated activation-induced cell death. Nature 2005;434:88-93.

34. Renshaw SA, Parmar JS, Singleton V, et al. Acceleration of human neutrophil apoptosis by TRAIL. J Immunol 2003;170:1027-33.

35. Jo M, Kim TH, Seol DW, et al. Apoptosis induced in normal human hepatocytes by tumor necrosis factorrelated apoptosis-inducing ligand. Nat Med 2000;6:564-7.

(English Language Editor: A. Muijlwijk) 

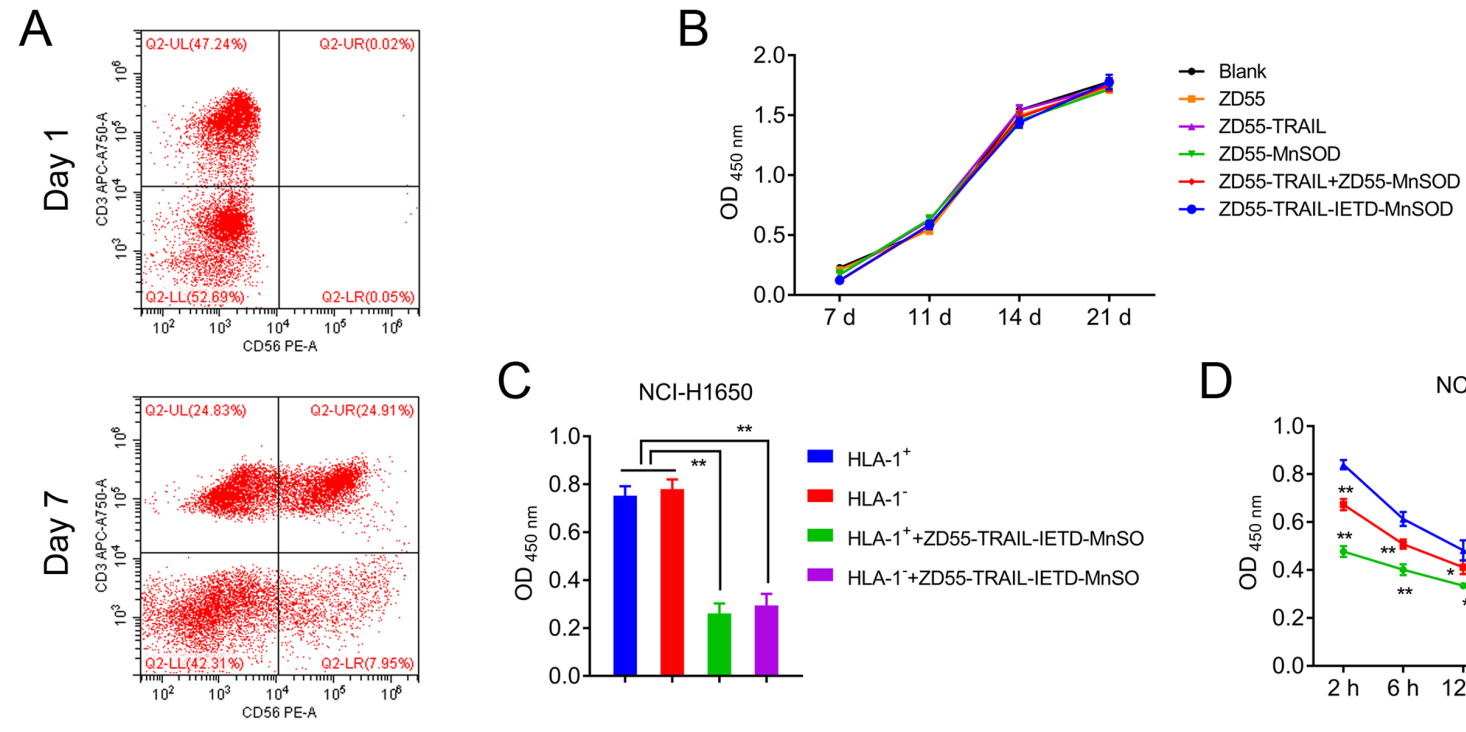

D $\quad \mathrm{NCl}-\mathrm{H} 1650$
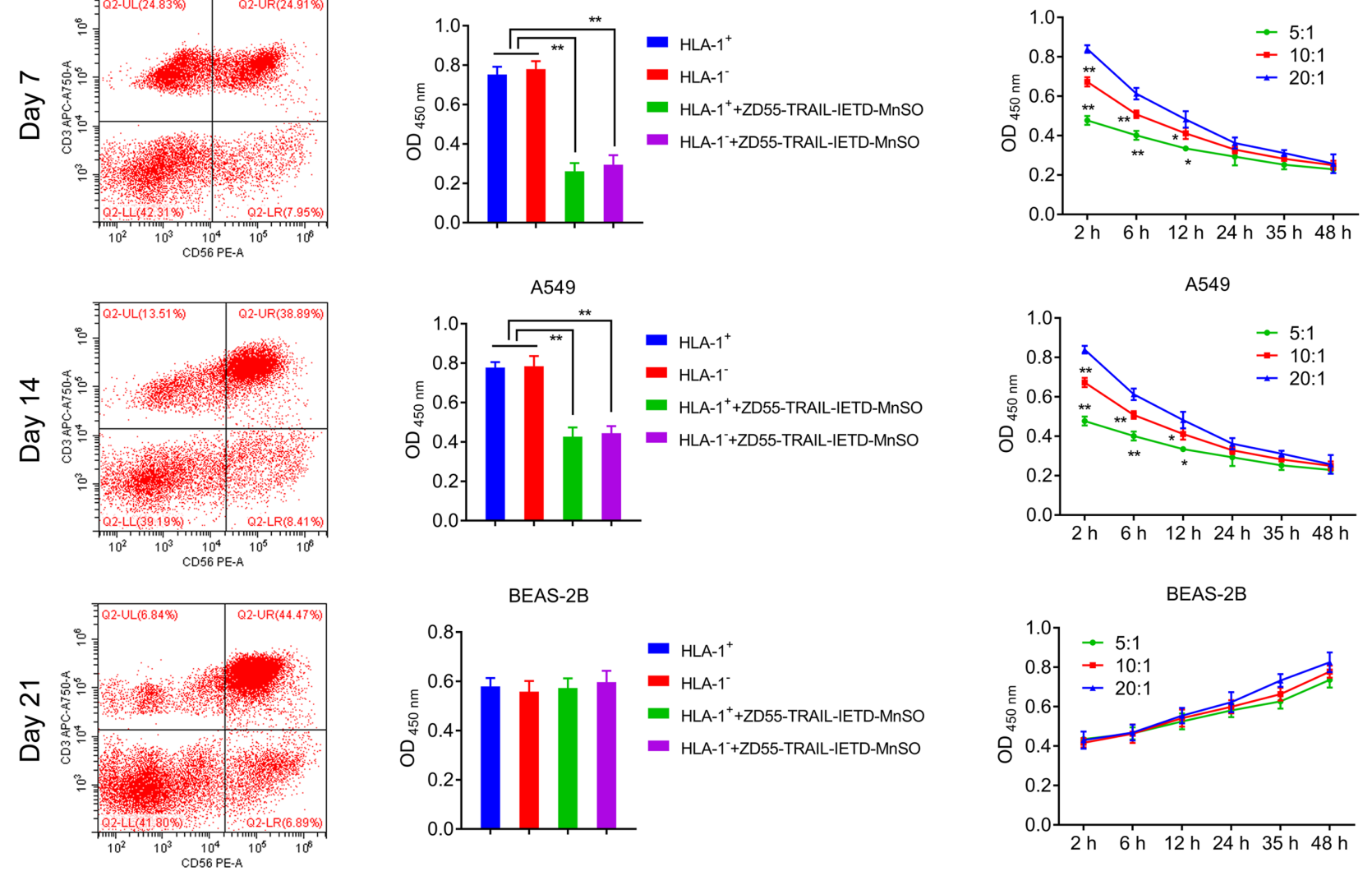

Figure S1 The population and cytotoxicity of CD3+CD56+ CIK cells, and the effect on the proliferation of lung cancer cell lines NCI-H1650 and A549, and human lung epithelial cells BEAS-2B. (A) CIK cells were stained for CD3 and CD56, and the population of corresponding CIK cells was calculated; (B) cell viability of CIK cells harboring oncolytic adenoviruses ZD55-EGFP, ZD55-TRAIL, ZD55MnSOD, ZD55-TRAIL+ZD55-MnSOD, and ZD55-TRAIL-IETD-MnSOD was determined at 7, 11, 14, and 21 days post-transfection; (C) cytopathic effects of CIK cells expressing the TRAIL-IETD-MnSOD fusion gene on the proliferation of HLA-I ${ }^{+}$and HLA-I cells of NCI-H1650, A549, and BEAS-2B; (D) cytopathic effects of CIK cells expressing TRAIL-IETD-MnSOD fusion gene on the proliferation of NCI-H1650, A549, and BEAS-2B at different effector-to-target (E:T) ratios in co-cultivated cells by CCK8. ${ }^{*} \mathrm{P}<0.05$ and ${ }^{* *} \mathrm{P}<0.01$ indicated a statistically significant difference between 2 groups. 